ARTICLE

Received 11 Dec 2015 | Accepted 15 Jun 2016 | Published 12 Aug $2016 \quad$ DOl: 10.1038/ncomms12261

\title{
Superior room-temperature ductility of typically brittle quasicrystals at small sizes
}

Yu Zou ${ }^{1} \dagger$, Pawel Kuczera ${ }^{2}$, Alla Sologubenko1, Takashi Sumigawa ${ }^{3}$, Takayuki Kitamura ${ }^{3}$, Walter Steurer ${ }^{2}$ \& Ralph Spolenak ${ }^{1}$

The discovery of quasicrystals three decades ago unveiled a class of matter that exhibits long-range order but lacks translational periodicity. Owing to their unique structures, quasicrystals possess many unusual properties. However, a well-known bottleneck that impedes their widespread application is their intrinsic brittleness: plastic deformation has been found to only be possible at high temperatures or under hydrostatic pressures, and their deformation mechanism at low temperatures is still unclear. Here, we report that typically brittle quasicrystals can exhibit remarkable ductility of over $50 \%$ strains and high strengths of $\sim 4.5 \mathrm{GPa}$ at room temperature and sub-micrometer scales. In contrast to the generally accepted dominant deformation mechanism in quasicrystals-dislocation climb, our observation suggests that dislocation glide may govern plasticity under high-stress and low-temperature conditions. The ability to plastically deform quasicrystals at room temperature should lead to an improved understanding of their deformation mechanism and application in small-scale devices.

\footnotetext{
${ }^{1}$ Department of Materials, Laboratory for Nanometallurgy, ETH Zurich, Vladimir-Prelog-Weg 5, CH-8093 Zurich, Switzerland. ${ }^{2}$ Department of Materials, Laboratory of Crystallography, ETH Zurich, Vladimir-Prelog-Weg 5, CH-8093 Zurich, Switzerland. ${ }^{3}$ Department of Mechanical Engineering and Science, Graduate School of Engineering, Kyoto University, Nishikyo-ku, Kyoto 615-8540, Japan. † Present address: Department of Mechanical Engineering, Massachusetts Institute of Technology, 77 Massachusetts Avenue, Cambridge, Massachusetts 02139, USA. Correspondence and requests for materials should be addressed to Y.Z. (email: yuzou@mit.edu) or to R.S. (email: ralph.spolenak@mat.ethz.ch).
} 
$\mathrm{n}$ materials science, plasticity describes the non-reversible deformation of a solid in response to applied forces and determines the ability of a material to change its shape permanently without breaking. Regular crystalline materials, including most metals and ceramics, are generally plastically deformed through dislocation motion ${ }^{1}$ or twinning ${ }^{2}$. The plasticity of amorphous solids, such as metallic glasses, is based on the formation and propagation of shear bands ${ }^{3}$. In quasicrystals ${ }^{4}$, despite their lack of periodicity, plastic deformation can also be achieved by dislocation activities ${ }^{5}$. In contrast to the situation in periodic crystals, every movement of a dislocation in a quasicrystal creates a cloud behind, which is called phason fault ${ }^{6}$. As a consequence, the dislocation motion gets hindered and the material appears brittle. Although a great variety of quasicrystals have been synthesized ${ }^{7,8}$, and some have even been discovered in nature ${ }^{9}$, and found to be technologically interesting $^{10-13}$ and useful ${ }^{14}$, only few of them can be found in applications so far, mainly limited by their poor ductility and formability at room temperature. Hence, improving the room-temperature ductility of quasicrystals is not only of academic interest but also essential for technological applications.

Early studies of the plastic deformation of quasicrystals focused on an easily grown icosahedral quasicrystal, i-Al-Pd-Mn, in the high-temperature regime above $\sim 600^{\circ} \mathrm{C}$ ( $\sim 70 \%$ of its melting temperature). These studies demonstrated that the plastic deformation of i-Al-Pd-Mn was dominated by dislocation climb-with the Burgers vector out of the plane of dislocation motion, rather than dislocation glide-with the Burgers vector restricted in the plane of dislocation motion ${ }^{15}$. It is generally

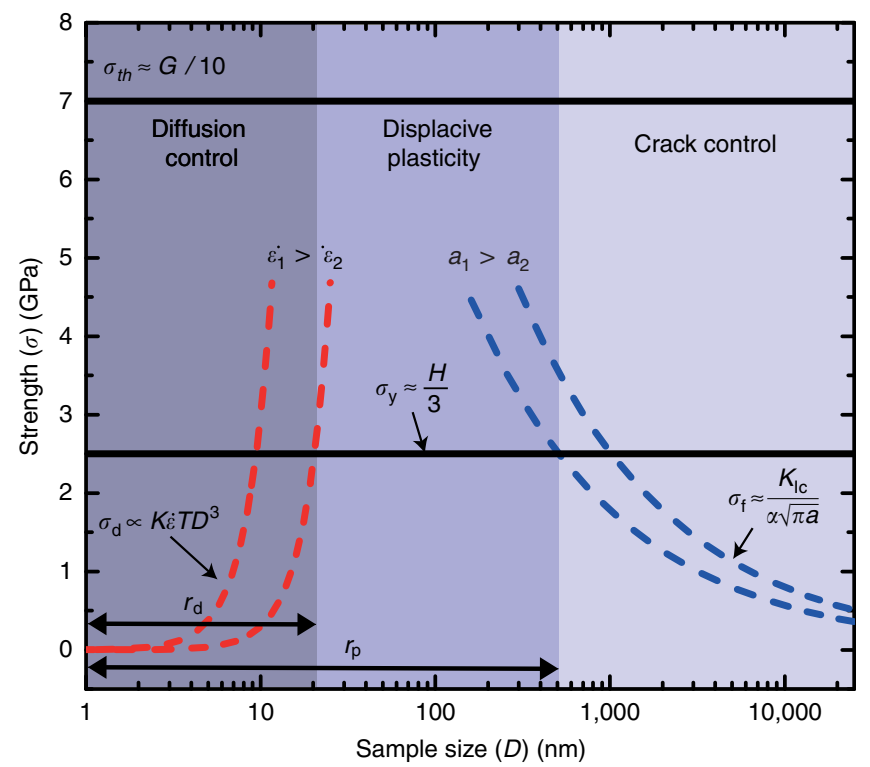

Figure 1 | Deformation map for small-scale i-Al-Pd-Mn quasicrystals.

Semi-quantitative predictions for room temperature deformation. If $D>r_{p}$, defined as the intersection of the fracture strength, $\sigma_{f}$ (the blue dashed lines), and the yield strength, $\sigma_{\mathrm{y}}$ (the black solid line), the material fails by cracking without notable plasticity, following the Griffith's criterion ${ }^{37}$, $\sigma_{\mathrm{f}}=K_{\mathrm{l} d} /\left[\alpha(\pi a)^{-1 / 2}\right]$ with $K_{\mathrm{lc}}$ the fracture toughness of the material, $\alpha$ a geometrical parameter on the order of unity and $a$ the size of pre-existing cracks or flaws. The $\sigma_{\mathrm{f}}$ shows a smaller-is-stronger trend. If $D<r_{\mathrm{d}}$, defined as the intersection of $\sigma_{\mathrm{y}}$ and $\sigma_{\mathrm{d}}$, the diffusion governs the strength, following $\sigma_{\mathrm{d}} \propto K \dot{\varepsilon} T D^{3}$ with $K$, surface diffusivity, $\dot{\varepsilon}$, strain rate, and $T$, temperature. The $\sigma_{\mathrm{d}}$ shows a smaller-is-weaker phenomenon. In between $r_{\mathrm{p}}$ and $r_{\mathrm{d}}$, the curves of $\sigma_{\mathrm{f}}, \sigma_{\mathrm{d}}$ and $\sigma_{\mathrm{y}}$ are crossed and define a zone controlled by displacive deformation. The size range of this zone may vary by flaw sizes and strain rates, as illustrated. believed that dislocation climb is a much easier deformation mode in quasicrystals than dislocation glide ${ }^{16}$. Although there are some hints that the glide motion may be possible in lowtemperature conditions as suggested by numerical simulations ${ }^{17}$ or under high hydrostatic pressures ${ }^{18}$, the required stress to activate glide is extremely high, on the order of $1 / 10$ of its shear modulus-a stress level generally leading to fracture without showing any ductility. It has been a long-standing question concerning the deformation mechanism in quasicrystals at room temperature. Despite several investigators have sought to explore the plastic deformation of quasicrystals at or near room temperature using indentation or by confining gas or solid pressures ${ }^{19-22}$, so far there has been no common conclusion: the explanations include shear banding similar to metallic glasses ${ }^{23}$, phase transformation ${ }^{24,25}$, grain-boundary glide $^{21}$, pure dislocation climb $^{22}$, dislocation climb dominant ${ }^{26}$ and crystallization ${ }^{27}$. Therefore, one has to conclude that the plastic deformation of quasicrystals under a wide range of temperatures and pressures has been poorly understood-much in contrast to crystalline and amorphous solids. Two fundamental questions are still open: can steady-state plastic deformation be achieved at room temperature? If so, what is the underlying deformation mechanism?

Unveiling room-temperature plasticity in quasicrystals hence relies on a new method to suppress fracture before plastic yielding in a simple loading experiment. Our strategy is to increase the fracture strength over the yield strength in a quasicrystal by reducing the sample size. Although similar methods have been explored for other brittle materials such as ceramics ${ }^{28}$ and metallic glasses ${ }^{29,30}$, it has, to our knowledge, not previously been reported for quasicrystals-a large family of unusual solids. In this study, we demonstrate a brittle-to-ductile transition in quasicrystals at room temperature due to a sample size reduction-a submicron-sized quasicrystal pillar exhibits superior ductility at room temperature. Furthermore, we suggest that dislocation glide may control the plastic deformation of quasicrystals at room temperature and attempt to shed light on the underlying deformation mechanism in the low-temperature regime.

\section{Results}

A model to predict brittle-to-ductile transition. To estimate at what size range a typically brittle quasicrystal may become ductile, we compared the different deformation mechanisms as a function of the sample size: dislocation activities, crack propagation $^{31}$ and mass transport by diffusion ${ }^{32}$. We identified three deformation regimes: cracking-controlled, displacivedeformation-controlled (dislocations or shear bands) and diffusion-controlled, as illustrated in Fig. 1. We estimated the critical size, $r_{\mathrm{p}}$, for the brittle-to-ductile transition to be $\sim 500 \mathrm{~nm}$, and the size of the diffusion-controlled zone, $r_{\mathrm{d}}$, to be around $10 \mathrm{~nm}$ (see the detailed analysis in Methods section at the end of the article). Our targeted sample size to attain steady-state plasticity thus falls in a range from $\sim 100$ to $\sim 500 \mathrm{~nm}$.

Micro-compression of small-sized quasicrystal pillars. In our experiments, we compressed single-quasicrystalline i-Al-Pd-Mn pillars with diameters ranging from $\sim 1.8 \mu \mathrm{m}$ to $\sim 150 \mathrm{~nm}$. We observed a brittle-to-ductile transition with the critical pillar diameter between 510 and $350 \mathrm{~nm}$ (Fig. 2a): the $1.8-\mu \mathrm{m}$ pillar exhibits a catastrophic failure at $\sim 3 \%$ compressive strain; the 870 - and 510-nm pillars show cracks at about $45^{\circ}$ along the loading direction, failing at $\sim 6 \%$ strain; when the pillar diameter is below $500 \mathrm{~nm}$, the pillars present significantly improved ductility with compressive strains over $50 \%$ and without any cracking. The $400-$ and $200-\mathrm{nm}$ pillars clearly show 

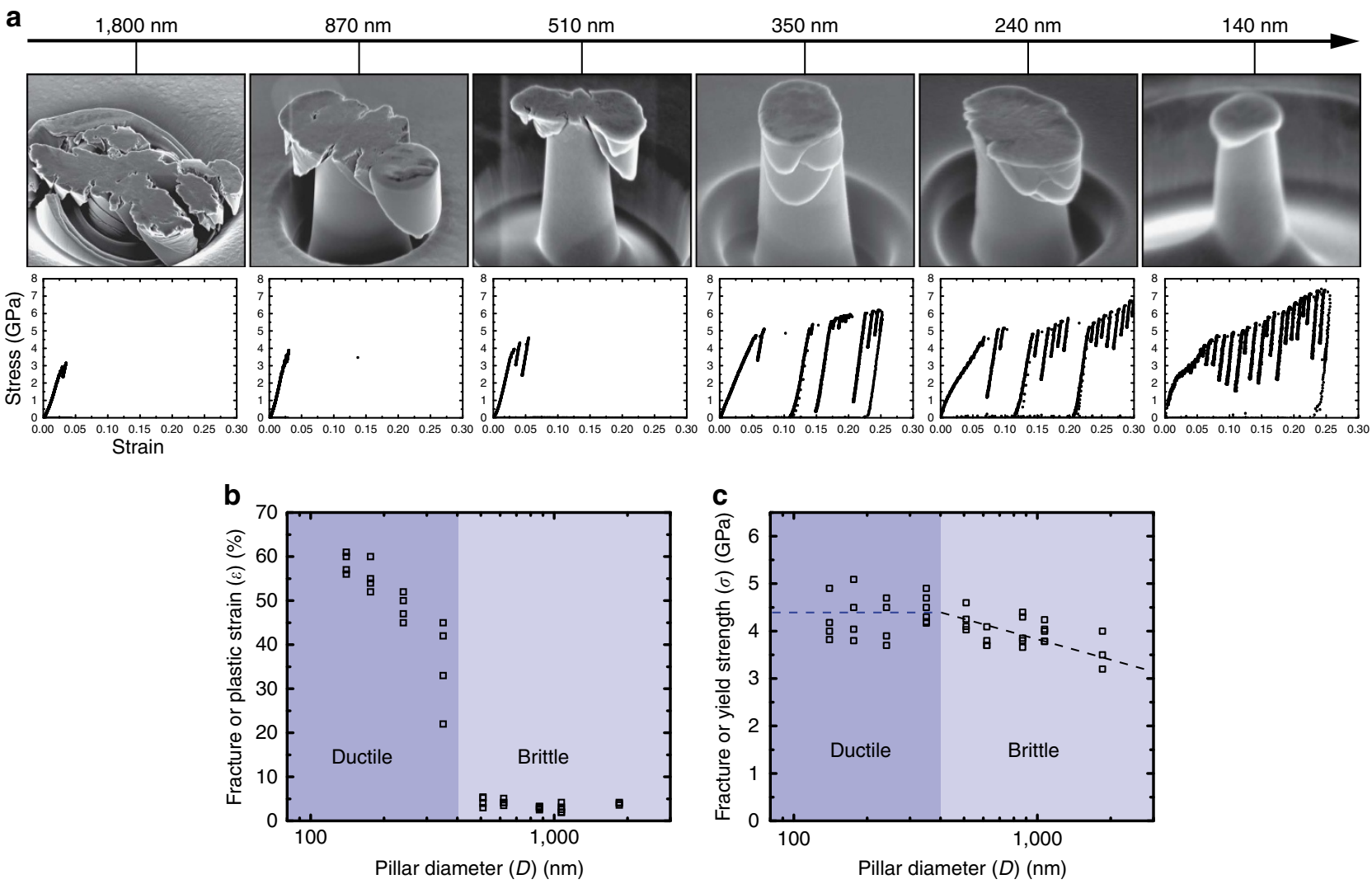

Figure 2 | Micro-compression of single-quasicrystalline i-Al-Pd-Mn pillars. Pillar diameters range from $\sim 2 \mu \mathrm{m}$ to $\sim 150 \mathrm{~nm}$. (a) Typical SEM images of the post-deformed pillars, showing a brittle-to-ductile transition with the critical size between 350 and $510 \mathrm{~nm}$. The corresponding engineering stress-strain curves are presented below. (b) The fracture strain or plastic strain as a function of the pillar diameter, indicating a brittle-to-ductile transition. (c) The fracture strength or yield strength as a function of the pillar diameter. In the brittle regime, the strength increases slightly with decreasing the pillar diameter; in the ductile regime, the strength is almost independent of the sample size.
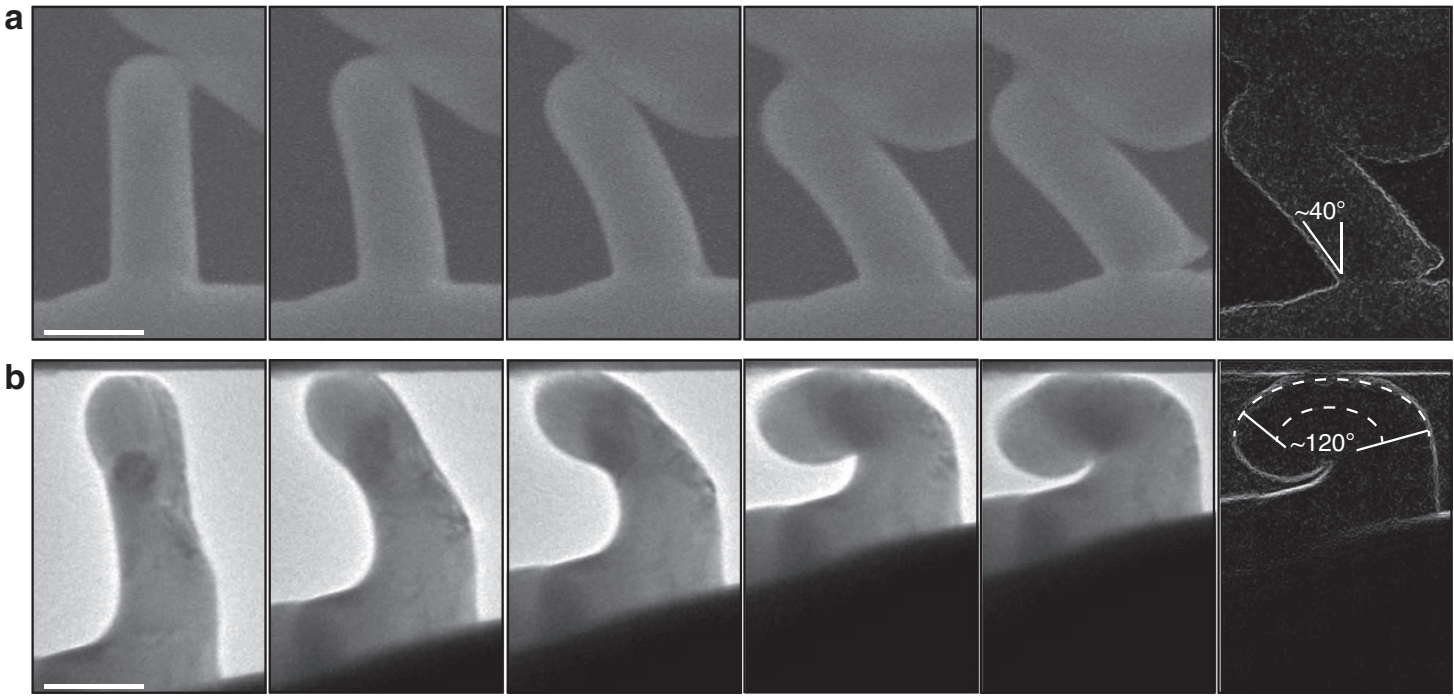

Figure 3 | In situ SEM and TEM of i-Al-Pd-Mn pillars during bending tests. (a) SEM snapshots captured during the bending test of a pillar with the diameter of $\sim 300 \mathrm{~nm}$. An initial crack occurs near the pillar base at the bending angle of $\sim 20-30^{\circ}$ and eventual fracture happens at the bending angle of $\sim 40^{\circ}$. (b) TEM snapshots during bending tests on a pillar in the diameter of $\sim 100 \mathrm{~nm}$, showing a homogenous deformation without any fracture, and the maximum tensile strain at the pillar centre estimated to be over 50\%. Scale bars, $300 \mathrm{~nm}$ (a) and $100 \mathrm{~nm}(\mathbf{b})$.

deformation bands, while the 140 -nm pillar reveals the deformation localized at the upper part of the pillar. All the corresponding stress-strain curves exhibit a displacement-burst phenomenon (Fig. 2a), which is generally observed in metals ${ }^{33,34}$ and metallic glasses ${ }^{29,30}$. The 140 - and 240 -nm pillars exhibit earlier plastic yielding than the other pillars, which could be due to localized deformation on the pillar top region or the lateral friction between the indenter tip and the top surface of the pillar. 

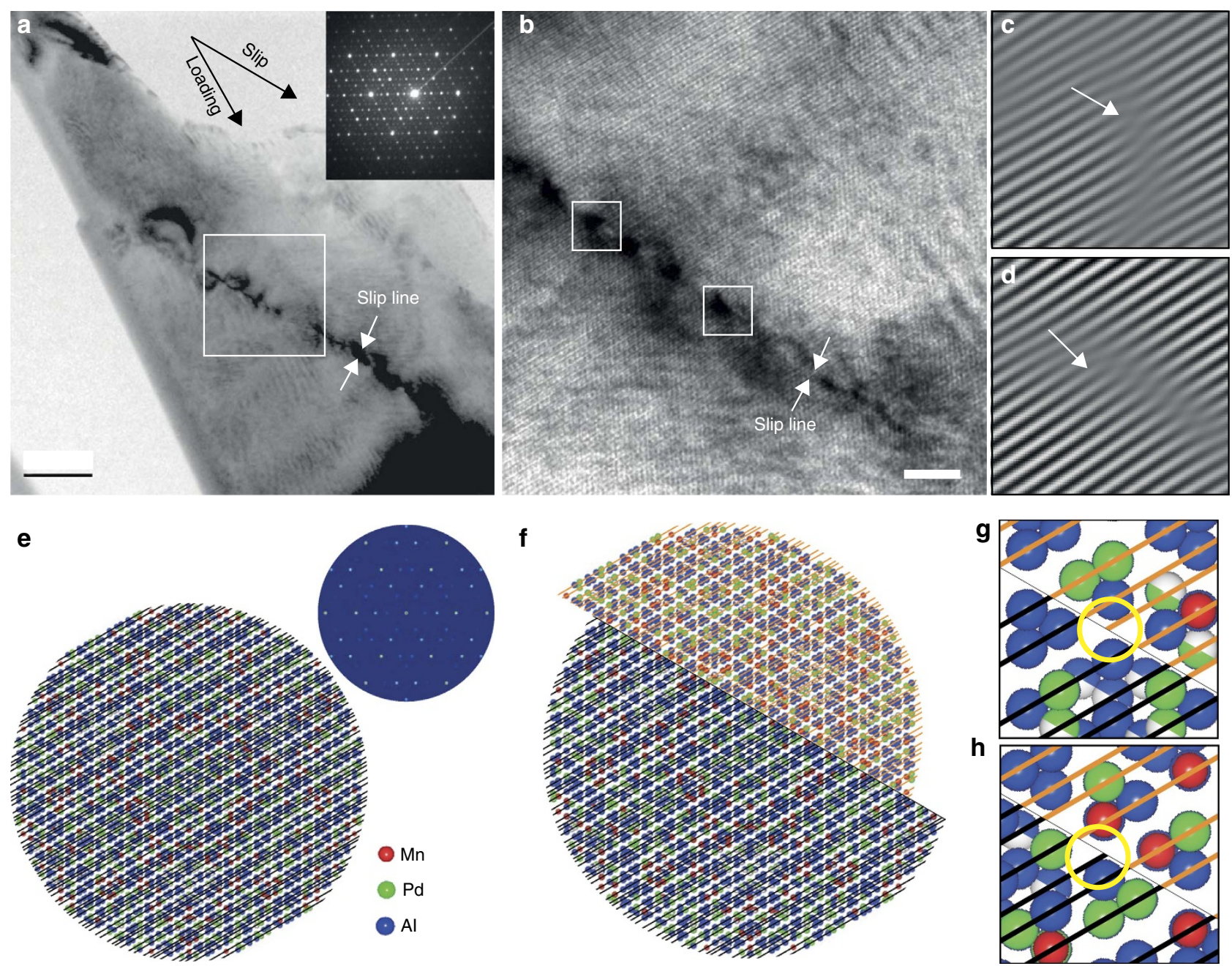

Figure 4 | Locally deformed region in i-Al-Pd-Mn pillar. TEM images observed along a threefold axis. (a) A typical bright-field TEM image showing a narrow and straight band traversing the pillar and the corresponding electron diffraction pattern. The loading direction is along a twofold axis and the slip direction is along another twofold axis. (b) The high-resolution TEM image shows the deformation band with a thickness of $\sim 2-5 \mathrm{~nm}$ and strain contrast modulations along the line (periodic dark regions along the band). The rest of the area is nearly defect-free. (c,d) The inverse Fourier transformation of the regions marked in $\mathbf{b}$, emphasizing the very localized and periodic lattice distortions along the deformation band. The inserted fringes are indicated by arrows. (e) The atomic model of $\mathrm{i}-\mathrm{Al}-\mathrm{Pd}-\mathrm{Mn}$ projected along the threefold axis with its calculated diffraction pattern to be compared with the experimental one in $\mathbf{a}$, before the deformation. (f) A schematic view in projection of the model after shear deformation, with the same loading and slip directions as shown in $\mathbf{b}$. (g,h) The local mismatches between the quasi-lattice planes, where the strain is concentrated, and correlating (c) and (d) (the black and orange lines indicate quasi-lattice planes and the circles indicate dislocations). Scale bars, $50 \mathrm{~nm}$ (a) and $10 \mathrm{~nm}$ (b).

Here, the flow stresses after the first displacement bursts were used to give a best estimation of their yield strengths. How the fracture strain or maximum plastic strain changes by decreasing the sample size also demonstrates the brittle-to-ductile transition between 510 and $350 \mathrm{~nm}$ (Fig. 2b). When the pillar diameter is smaller than $350 \mathrm{~nm}$, no cracking is observed in our experiments. Regarding the size dependence of strength, the fracture strength increases from $\sim 3.5$ to $\sim 4.5 \mathrm{GPa}$ with decreasing pillar diameters in the brittle regime, while the yield strength (the flow stress at the first displacement burst) is about $4.5 \mathrm{GPa}$ in the ductile regime (Fig. 2c).

In situ bending tests of small-sized quasicrystal pillars. Brittle materials usually show higher ductility in compression than tension. To examine the tensile ductility of the quasicrystal pillars but avoid the complex experimental setup of the tensile test for sub-micrometer-sized samples, we employed micro-bending tests to induce an asymmetrical stress distribution and compare the bending ductility of the pillars in different sizes. The in situ scanning electron microscopy (SEM) bending of a 300-nm pillar shows that the deformation localizes near the pillar base by necking. We detected that the crack forms at the bending angle of $\sim 20-30^{\circ}$, and eventually fails in a catastrophic feature at the bending angle of $\sim 40^{\circ}$ (Fig. 3a). The in situ transmission electron microscopy (TEM) bending of a $100-n m$ pillar shows in a rather homogenous deformation without any cracking and fracture (Fig. 3b). The longitudinal tensile strain near the pillar centre is estimated to be over $50 \%$. The strain bands' motion during the tests implies dislocation activity during the deformation (Supplementary Fig. 1).

TEM characterization and diffraction simulations. A representative bright-field TEM image reveals the upper part of a deformed pillar along a threefold axis (Fig. 4a). We find a slip line through the pillar and a step at the pillar edge. The loading direction is along a twofold axis and the slip plane contains another twofold axis. The high-resolution TEM image shows a very narrow band of $\sim 2-5 \mathrm{~nm}$ in thickness. Along the band, 


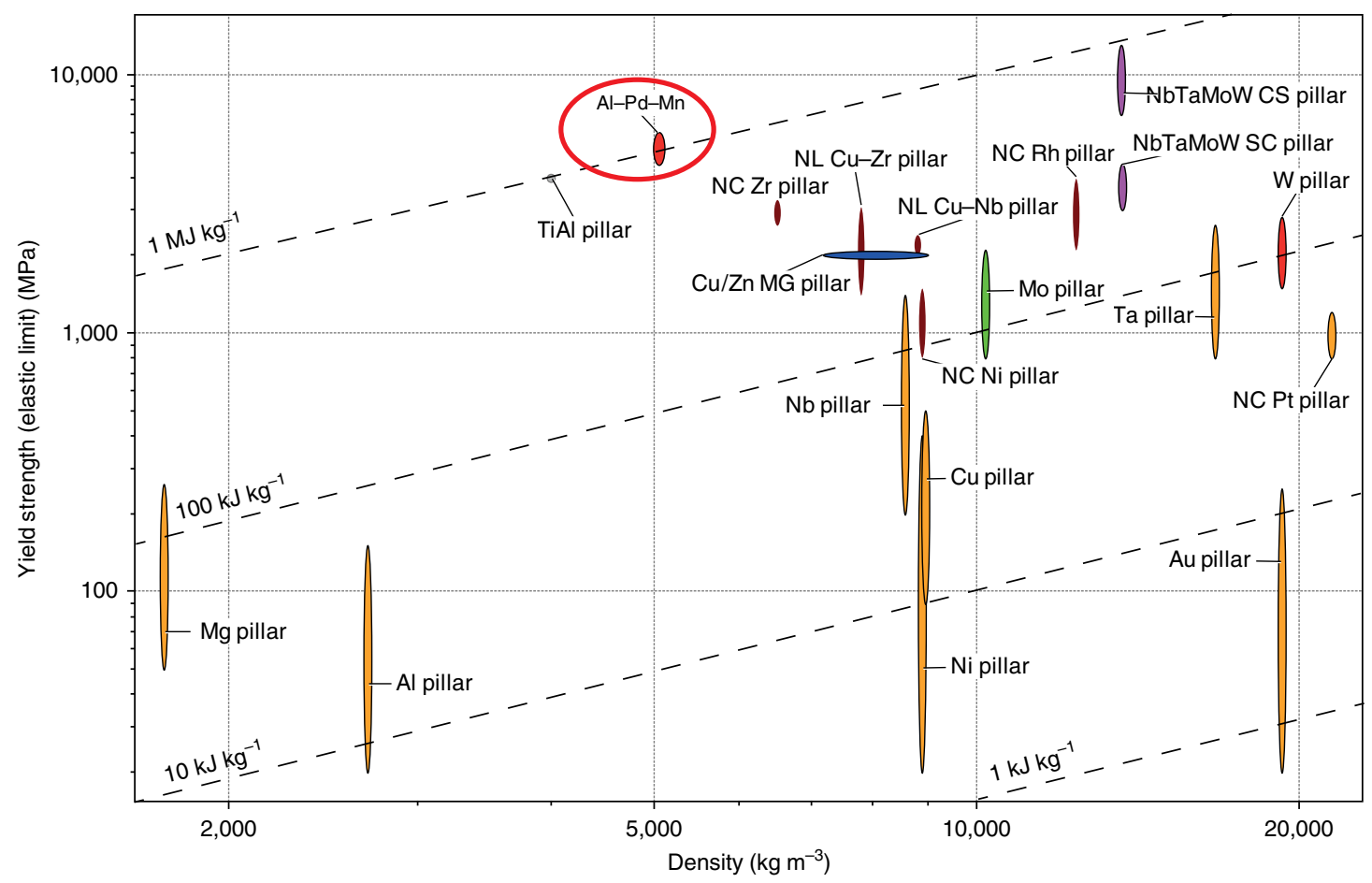

Figure 5 | Strength comparison with other metallic and metallic-glass pillars. Ashby map (designed with CES EduPack 2014) of yield strength versus density, indicating that i-Al-Pd-Mn quasicrystal pillars exhibit, to our knowledge, the highest specific strength or the strength-to-density ratio (CS, single crystalline; NC, nanocrystalline; NL, nanolamellar; MG, metallic glass). The strength levels of i-Al-Pd-Mn quasicrystal pillars are from Fig. 2c. Literature data for pillar strengths: pure metals $\mathrm{Au}^{34,44}, \mathrm{Al}^{45}, \mathrm{Ni}^{46}, \mathrm{Cu}^{47}, \mathrm{Nb}, \mathrm{Ta}, \mathrm{Mo}$ and $\mathrm{W}^{48,49}$ and $\mathrm{Mg}^{50}, \mathrm{TiAl}^{51}$, nanocrystalline ( $\mathrm{nc}$ ) $\mathrm{Cu}^{52}, \mathrm{Ni}^{53}, \mathrm{Ni}^{-\mathrm{W}^{54}}, \mathrm{Pt}^{55}$ and $\mathrm{Rh}^{56}$ pillars, NbTaMoW high-entropy alloys ${ }^{57,58}$, and metallic glasses (for example, $\mathrm{Cu}$ - and $\mathrm{Zn}$-based ones ${ }^{59}$ ).

there are strain-contrast modulations with a nearly equal distance of $\sim 2-5 \mathrm{~nm}$ and the area surrounding deformation band is nearly defect-free. (Fig. 4b; Supplementary Fig. 2). We do not observe any evidence of melting, crystallization, phase transformation or cracking that was used to explain room-temperature deformation in quasicrystals. Different from the deformation bands formed in $\mathrm{i}-\mathrm{Al}-\mathrm{Pd}-\mathrm{Mn}$ under hydrostatic pressures and at room temperature ${ }^{18}$, the bands observed here are much narrower and contain a much lower defect density. Using the inverse Fourier transformed images (Fig. 4c,d from the boxed areas in Fig. 4b), we identify a few inserted fringes along the deformation band. These inserted fringes indicate the distortions caused by dislocations. Together with the sharp deformation band and the step at the pillar edge (Fig. 4a), the fringes suggest that their Burgers vectors may contain the components along the slip or shear direction-dislocation glide might have occurred. Our atomic model of i-Al-Pd-Mn quasicrystal matches the orientation of the sample before and after deformation, respectively (Fig. 4e,f). Along the slip line shown in Fig. 4f, we can identify the mismatch region generated by the dislocation glide due to the local shear between the quasi-lattice planes (Fig. 4g,h). Such discontinuous quasi-lattice planes could be interpreted as dislocations with Burgers vector along the slip direction, which compares to the fringe patterns in Fig. 4c,d. The strain contrast shown in Fig. $4 \mathrm{~b}$ could be attributed to strain fields of the dislocations or related phason faults left behind. This is a strong indication that the plasticity of quasicrystals at room temperature can be dominated by dislocation glide.

\section{Discussion}

The results shown in Figs 2 and 3 confirm that i-Al-Pd-Mn pillars are capable of both excellent ductility (compressive and tensile) and maintaining high strength when the pillar diameter is below about $500 \mathrm{~nm}$. To our knowledge, this result has never been reported for quasicrystals before. The quasicrystal fine-scale pillars exhibit minor size dependence of strength and a deformation morphology with wavy features (see high-resolution SEM images in Supplementary Fig. 3), which is more similar to metallic glasses ${ }^{29,30}$ than to metals ${ }^{35}$. Nevertheless, we show that the quasicrystal plasticity at room temperature is still controlled by dislocation mechanisms.

Although in quasicrystals climb leads to the removal or insertion of so-called 'worms' without overlaps or open spaces ${ }^{15}$, this process requires thermal activation. At room temperature, the atomic diffusion in quasicrystals is generally believed to be inhibited. Dislocation glide, however, may be active and even dominate under high-stress and low-temperature conditions, generating a high density of heavily distorted zones in the wake of the dislocation glide. The approach of reducing sample size to enhance the ductility of otherwise brittle quasicrystals may pave way to fundamentally understand the deformation mechanism of quasicrystals at room temperature, possibly at even lower temperatures and for all the other types of quasicrystals ${ }^{36}$.

Towards technological applications, fine-scale quasicrystals are attractive not only due to combining high strength with ductility but also because they offer extraordinary specific strength (strength divided by density or elastic energy density, $\sim 1 \mathrm{MJ} \mathrm{kg}^{-1}$ ) among metallic micro/nano-pillars reported to date (Fig. 5), which might be used to store elastic energy. Small dimensional quasicrystals having superior strength and ductility, together with their interesting functional properties, may also enable components that are both structurally and functionally useful in micro- or nano-electromechanical systems. While much work remains to optimize their properties, our observation of superior room-temperature ductility in quasicrystals motivates further fundamental and technological exploration. 


\section{Methods}

Sample preparation and characterization. An initial compact of composition $\mathrm{Al}_{70} \mathrm{Pd}_{21.5} \mathrm{Mn}_{8.5}$ was prepared from pure metals (Al 99.9999\%, Pd 99.9\%, Mn $99.95 \%$ ). The sample was pre-alloyed in an arc furnace, and subsequently placed in an $\mathrm{Al}_{2} \mathrm{O}_{3}$ crucible and sealed in a quartz glass ampoule under an $\mathrm{Ar}$ atmosphere. The heat treatment consisted of the following steps: heating to $1323 \mathrm{~K}$ (above its melting temperature), slow cooling to $1083 \mathrm{~K}$ at the rate of $30 \mathrm{~K} \mathrm{~h}^{-1}$, annealing at $1,083 \mathrm{~K}$ for $150 \mathrm{~h}$, and subsequent quenching in water. The composition of the resulting sample was confirmed using energy dispersive X-ray spectroscopy. The X-ray powder diffraction pattern (Supplementary Fig. 4) and TEM diffraction patterns (Supplementary Fig. 5) indicate that the resulting sample is a single-phase icosahedral quasicrystal, which is comparable to that in literature ${ }^{60}$.

The prepared i-Al-Pd-Mn was thermodynamically stable with an average grain size of about $300 \mu \mathrm{m}$ and was also highly isotropic. We fabricated single-quasicrystalline pillars, in cylindrical shapes, from a coarse grain in a well-polished i-Al-Pd-Mn sample using a FIB system (Helios Nanolab 600i, FEI): a coarse milling condition of $30 \mathrm{kV}$ and $80 \mathrm{pA}$ and a final milling condition of $5 \mathrm{kV}$ and $7 \mathrm{pA}$. The diameters of the FIB-milled pillars are in the range of $\sim 150 \mathrm{~nm}$ to $\sim 2 \mu \mathrm{m}$ and the aspect ratios are $\sim 3.0-4.5$. A taper of $2-3^{\circ}$ was generally observed and the top diameter of the pillar was chosen to calculate stress.

Micro-mechanical testing. We used the nanoindenter (Hysitron Inc., USA) with a diamond flat-punch tip ( $5 \mu \mathrm{m}$ in diameter, Synton-MDP, Switzerland) to compress the pillars in a displacement control mode and the strain rate of $2 \times 10^{-3} s^{-1}$ by feedback mechanism. At least four pillars for each size were compressed. The deformed pillars were imaged using a high-resolution SEM (Magellan, FEI). For the post-mortem TEM characterization, the deformed pillars were thinned down to a lamella by ion milling, lift-out, thinning and polishing in the FIB system. Their cross-sections were then examined using a TEM (Tecnai F30, FEI, operated at $300 \mathrm{kV}$ ). In situ SEM and TEM bending tests were carried out using a nano-manipulator (Kleindiek, Germany) fitted to a SEM (Hitachi SU 8200) and an indenter holder (Nanofactory Instruments AB, SA2000N) fitted to a TEM (JEOL JEM-2100), respectively, with a displacement rate of $\sim 5 \mathrm{~nm} \mathrm{~s}^{-1}$.

Prediction of the brittle-to-ductile transition. In a brittle material, the fracture strength, $\sigma_{\mathrm{f}}$, follows the Griffith's criterion ${ }^{37}$, as $\sigma_{\mathrm{f}}=K_{\mathrm{Ic}} /\left[\alpha(\pi a)^{1 / 2}\right]$ with $K_{\mathrm{Ic}}$ the fracture toughness of the material, $\alpha$ a geometrical parameter on the order of unit and $a$ the size of pre-existing cracks or flaws. Statistically, larger samples are more likely to contain larger flaws, or weaker links, and consequently, smaller samples usually exhibit higher fracture strengths than the large ones-the size effect due to the Weibull statistics ${ }^{38}$. Because the fracture strength, $\sigma_{\mathrm{f}}$, cannot rise above the yield strength, $\sigma_{\mathrm{y}}$, below a certain length scale plastic flow may determine the strength. The intersection between the curves for $\sigma_{\mathrm{f}}$ and $\sigma_{\mathrm{y}}$ provides a critical size, $r_{\mathrm{p}}$, for a brittle-to-ductile transition, as illustrated in Fig. 1a. Assuming that the largest pre-existing cracks or flaws is one order of magnitude smaller than the sample dimension, we can obtain $r_{\mathrm{p}}$ of $\sim 500 \mathrm{~nm}$ for i-Al-Pd-Mn, using $\alpha, \sim 1$, $K_{\mathrm{Ic}}, \sim 1.25 \mathrm{MPa} \times m^{1 / 2}$ (ref. 39) and the hardness, $H, \sim 8.5 \mathrm{GPa}$ (ref. 39). However, further reduction of the sample size down to the nanometre scale leads to a significant increase of the surface-to-volume ratio, and surface diffusion may control the plastic flow, resulting in a reduced strength. In a relation similar to the Coble creep ${ }^{40}$, the diffusion strength, $\sigma_{\mathrm{d}}$, reflects a 'smaller-is-weaker' phenomenon. The crossover between $\sigma_{\mathrm{d}}$ and $\sigma_{\mathrm{y}}$ defines a diffusion-controlled zone with the length scale of $r_{\mathrm{d}}$ (Fig. 1a). Although it is difficult to calculate the exact value of $r_{\mathrm{d}}$ due to the lack of available literature data, recent studies on $\mathrm{Al}_{90} \mathrm{Fe}_{5} \mathrm{Ce}_{5}$ metallic glass ${ }^{41}$ and pure $\mathrm{Sn}^{42}$ demonstrate that diffusion controls plasticity below the sample sizes of $20 \mathrm{~nm}$ and $130 \mathrm{~nm}$, respectively, at a strain rate of $\sim 10^{-3} \mathrm{~s}^{-1}$ and room temperature. Hence, we estimate the $r_{\mathrm{d}}$ for i-Al-Pd-Mn as a few tens of nanometres (definitely smaller than $100 \mathrm{~nm}$ ), under similar experimental conditions. On the basis of this analysis, our targeted sample size to attain steady-state plasticity falls in a range from $\sim 100$ to $\sim 500 \mathrm{~nm}$.

Diffraction simulations. We used the Quiquandon-Gratias atomic model ( $\sim 70 \AA$ in diameter) of icosahedral Al-Pd-Mn ${ }^{43}$. We oriented the model along the threefold axis (high-magnification image in Supplementary Fig. 6), and calculated the diffraction pattern and compared with the experimental electron diffraction pattern. This agreement indicates that the orientation of our model matches the orientation of the sample.

Data availability. The data that support the findings of this study are available from the corresponding author upon request.

\section{References}

1. Johnston, W. G. \& Gilman, J. J. Dislocation velocities, dislocation densities, and plastic flow in lithium fluoride crystals. J. Appl. Phys. 30, 129-144 (1959).

2. Christian, J. W. \& Mahajan, S. Deformation twinning. Prog. Mater. Sci. 39, 1-157 (1995).

3. Greer, A. L., Cheng, Y. Q. \& Ma, E. Shear bands in metallic glasses. Mater. Sci. Eng. $R$ 74, 71-132 (2013).
4. Shechtman, D., Blech, I., Gratias, D. \& Cahn, J. W. Metallic phase with long-range orientational order and no translational symmetry. Phys. Rev. Lett. 53, 1951-1953 (1984).

5. Levine, D. et al. Elasticity and dislocations in pentagonal and icosahedral quasicrystals. Phys. Rev. Lett. 54, 1520-1523 (1985).

6. Socolar, J. E. S., Lubensky, T. C. \& Steinhardt, P. J. Phonons, phasons, and dislocations in quasi-crystals. Phys. Rev. B 34, 3345-3360 (1986).

7. Takakura, H., Gomez, C. P., Yamamoto, A., De Boissieu, M. \& Tsai, A. P. Atomic structure of the binary icosahedral $\mathrm{Yb}-\mathrm{Cd}$ quasicrystal. Nat. Mater. 6, 58-63 (2007).

8. Zeng, X. et al. Supramolecular dendritic liquid quasicrystals. Nature 428, 157-160 (2004).

9. Bindi, L., Steinhardt, P. J., Yao, N. \& Lu, P. J. Natural quasicrystals. Science 324 1306-1309 (2009).

10. Dubois, J. M. Properties- and applications of quasicrystals and complex metallic alloys. Chem. Soc. Rev. 41, 6760-6777 (2012).

11. Dolinsek, J. Electrical and thermal transport properties of icosahedral and decagonal quasicrystals. Chem. Soc. Rev. 41, 6730-6744 (2012).

12. Man, W., Megens, M., Steinhardt, P. J. \& Chaikin, P. M. Experimental measurement of the photonic properties of icosahedral quasicrystals. Nature 436, 993-996 (2005).

13. Deguchi, K. et al. Quantum critical state in a magnetic quasicrystal. Nat. Mater. 11, 1013-1016 (2012).

14. Dubois, J. M. Useful Quasicrystals (World Scientific, 2005)

15. Caillard, D., Mompiou, F., Bresson, L. \& Gratias, D. Dislocation climb in icosahedral quasicrystals. Scr. Mater. 49, 11-17 (2003).

16. Bonneville, J., Caillard, D. \& Guyot, P. in Dislocations and Plasticity of Icosahedral Quasicrystals: Dislocations in Solids (ed. Hirth, J. P.) Ch. 85 (Elsevier, 2008).

17. Dilger, C., Mikulla, R., Roth, J. \& Trebin, H. R. Simulation of shear stress in icosahedral quasicrystals. Philos. Mag. 75, 425-441 (1997).

18. Texier, M., Proult, A., Bonneville, J. \& Rabier, J. Microstructure of I-Al-Pd-Mn deformed at low and intermediate temperatures. Mater. Sci. Eng. A 387-389, 1023-1027 (2004).

19. Feuerbacher, M. et al. The plasticity of icosahedral quasicrystals. Mater. Sci. Eng. A 233, 103-110 (1997).

20. Kabutoya, E. et al. Plastic deformation of icosahedral Al-Pd-Mn single quasicrystals to large strains-I. Experiments. Philos. Mag. A 82, 369-377 (2002).

21. Wollgarten, M., Saka, H. \& Inoue, A. Microstructural investigation of the brittle-to-ductile transition in Al-Pd-Mn quasicrystals. Philos. Mag. A 79, 2195-2208 (1999).

22. Mompiou, F. \& Caillard, D. Plasticity of single-grain icosahedral Al-Pd-Mn quasi-crystals deformed at room temperature. Acta Mater. 52, 3613-3619 (2004).

23. Azhazha, V. et al. Structure and peculiarities of nanodeformation in $\mathrm{Ti}-\mathrm{Zr}-\mathrm{Ni}$ quasi-crystals. Philos. Mag. 84, 983-990 (2004)

24. Wu, J., Brien, V., Brunet, P., Dong, C. \& Dubois, J. Electron microscopy study of scratch-induced surface microstructures in an $\mathrm{Al}-\mathrm{Cu}-\mathrm{Fe}$ icosahedral quasicrystal. Philos. Mag. A 80, 1645-1655 (2000).

25. Texier, M., Thilly, L., Bonneville, J., Proult, A. \& Rabier, J. Shear experiments under confining pressure conditions of Al-Pd-Mn single quasicrystals. Mater. Sci. Eng. A 400-401, 311-314 (2005).

26. Texier, M., Joulain, A., Bonneville, J., Thilly, L. \& Rabier, J. Al-Pd-Mn icosahedral quasicrystal: deformation mechanisms in the brittle domain. Philos Mag. 87, 1497-1511 (2007).

27. Reibold, M., Belger, A., Mukhopadhyay, N. K., Gille, P. \& Paufler, P. The impact of nanoindentation at room temperature upon the real structure of decagonal AlCoNi quasicrystals. Phy. Status Solidi A 202, 2267-2276 (2005).

28. Ostlund, F. et al. Brittle-to-ductile transition in uniaxial compression of silicon pillars at room temperature. Adv. Funct. Mater. 19, 2439-2444 (2009).

29. Volkert, C., Donohue, A. \& Spaepen, F. Effect of sample size on deformation in amorphous metals. J Appl. Phys. 103, 083539-083536 (2008).

30. Jang, D. C. \& Greer, J. R. Transition from a strong-yet-brittle to a stronger-andductile state by size reduction of metallic glasses. Nat. Mater. 9, 215-219 (2010)

31. Lawn, B. Fracture of Brittle Solids 2nd edn (Cambridge Univ. Press, 1993).

32. Herring, C. Diffusional viscosity of a polycrystalline solid. J. Appl. Phys. 21, 437-445 (1950).

33. Dimiduk, D. M., Woodward, C., LeSar, R. \& Uchic, M. D. Scale-free intermittent flow in crystal plasticity. Science 312, 1188-1190 (2006).

34. Greer, J. R., Oliver, W. C. \& Nix, W. D. Size dependence of mechanical properties of gold at the micron scale in the absence of strain gradients. Acta Mater. 53, 1821-1830 (2005).

35. Uchic, M. D., Dimiduk, D. M., Florando, J. N. \& Nix, W. D. Sample dimensions influence strength and crystal plasticity. Science 305, 986-989 (2004).

36. Zou, Y., Kuczera, P., Steurer, W. \& Spolenak, R. Disappearance of plastic anisotropy in decagonal quasicrystals at small scales and room temperature. Extreme Mech. Lett.. doi:10.1016/j.eml.2016.02.005 (2016). 
37. Griffith, A. A. The phenomena of rupture and flow in solids. Phil. Trans. R. Soc. Lond. A 221, 163-198 (1921).

38. Weibull, W. A statistical distribution function of wide applicability. J. Appl. Mech. 13, 293-297 (1951)

39. Deus, C., Wolf, B. \& Paufler, P. On the orientation dependence of crack-like failure formation near indentations on an icosahedral Al-Pd-Mn quasicrystal. Philos. Mag. A 75, 1171-1183 (1997).

40. Coble, R. L. A model for boundary diffusion controlled creep in polycrystalline materials. J. Appl. Phys. 34, 1679-1682 (1963).

41. Luo, J., Wu, F., Huang, J., Wang, J. \& Mao, S. Superelongation and atomic chain formation in nanosized metallic glass. Phys. Rev. Lett. 104, 215503 (2010).

42. Tian, L., Li, J., Sun, J., Ma, E. \& Shan, Z. W. Visualizing size-dependent deformation mechanism transition in Sn. Sci. Rep. 3, 2113 (2013)

43. Quiquandon, M. \& Gratias, D. Unique six-dimensional structural model for Al-Pd-Mn and Al-Cu-Fe icosahedral phases. Phys. Rev. B 74, 214205 (2006).

44. Volkert, C. A. \& Lilleodden, E. T. Size effects in the deformation of sub-micron Au columns. Philos. Mag. 86, 5567-5579 (2006).

45. Kunz, A., Pathak, S. \& Greer, J. R. Size effects in Al nanopillars: single crystalline vs bicrystalline. Acta Mater. 59, 4416-4424 (2011).

46. Frick, C. P., Clark, B. G., Orso, S., Schneider, A. S. \& Arzt, E. Size effect on strength and strain hardening of small-scale [111] nickel compression pillars. Mater. Sci. Eng. A 489, 319-329 (2008).

47. Jennings, A. T., Burek, M. J. \& Greer, J. R. Microstructure versus size: mechanical properties of electroplated single crystalline $\mathrm{Cu}$ nanopillars. Phys. Rev. Lett. 104, 135503 (2010).

48. Schneider, A. S. et al. Correlation between critical temperature and strength of small-scale bcc pillars. Phys. Rev. Lett. 103, 105501 (2009).

49. Kim, J.-Y., Jang, D. \& Greer, J. R. Tensile and compressive behavior of tungsten, molybdenum, tantalum and niobium at the nanoscale. Acta Mater. 58, 2355-2363 (2010).

50. Lilleodden, E. Microcompression study of Mg (0001) single crystal. Scr. Mater. 62, 532-535 (2010).

51. Edalati, K. et al. Ultrahigh strength and high plasticity in TiAl intermetallics with bimodal grain structure and nanotwins. Scr. Mater. 67, 814-817 (2012).

52. Okamoto, N. L., Kashioka, D., Hirato, T. \& Inui, H. Specimen- and grain-size dependence of compression deformation behavior in nanocrystalline copper. Int. J. Plast. 56, 173-183 (2014).

53. Rinaldi, A., Peralta, P., Friesen, C. \& Sieradzki, K. Sample-size effects in the yield behavior of nanocrystalline nickel. Acta Mater. 56, 511-517 (2008).

54. Jang, D. \& Greer, J. R. Size-induced weakening and grain boundary-assisted deformation in $60 \mathrm{~nm}$ grained Ni nanopillars. Scr. Mater. 64, 77-80 (2011).

55. Gu, X. W. et al. Size-dependent deformation of nanocrystalline Pt nanopillars. Nano Lett. 12, 6385-6392 (2012).

56. Alshehri, O., Yavuz, M. \& Tsui, T. Manifestation of external size reduction effects on the yield point of nanocrystalline rhodium using nanopillars approach. Acta Mater. 61, 40-50 (2013).

57. Zou, Y., Ma, H. \& Spolenak, R. Ultrastrong ductile and stable high-entropy alloys at small scales. Nat. Commun. 6, 7748 (2015).
58. Zou, Y., Maiti, S., Steurer, W. \& Spolenak, R. Size-dependent plasticity in an $\mathrm{Nb}_{25} \mathrm{Mo}_{25} \mathrm{Ta}_{25} \mathrm{~W}_{25}$ refractory high-entropy alloy. Acta Mater. 65, 85-97 (2014).

59. Chen, C. Q., Pei, Y. T. \& De Hosson, J. T. M. Effects of size on the mechanical response of metallic glasses investigated through in situ TEM bending and compression experiments. Acta Mater. 58, 189-200 (2010).

60. Tsai, A. P., Inoue, A., Yokoyama, Y. \& Masumoto, T. Stable icosahedral Al-Pd-Mn and Al-Pd-Re alloys. Mater. Trans. JIM 31, 98-103 (1990).

\section{Acknowledgements}

We thank S. Takeuchi (Tokyo University of Science) and K. Edagawa (The University of Tokyo) for their helpful discussion, T. Yukishita and S. Ashida (Kyoto University) for their help in the bending tests, ScopeM (ETH Zurich) for supplying electron microcopy facility and A. Evans for proofreading. Y.Z. acknowledges the financial supports through the Swiss National Science Foundation (SNF Grants: 200021_143633 and P2EZP2_165278) and the JSPS strategic program (GR14103). T.S. and T.K. acknowledge the JSPS Grant (No. 25000012).

\section{Author contributions}

Y.Z., W.S. and R.S. created the idea and designed the project; P.K. prepared the bulk sample and did the simulation. Y.Z. carried out the nanomechanical testing; A.S. did the TEM analysis; T.S. and T.K. contributed the design of in situ SEM and TEM experiments. Y.Z. prepared the manuscript. W.S. and R.S. supervised the project. All the authors contributed to the discussion and the interpretation of the data.

\section{Additional information}

Supplementary Information accompanies this paper at http://www.nature.com/ naturecommunications

Competing financial interests: The authors declare no competing financial interests.

Reprints and permission information is available online at http://npg.nature.com/ reprintsandpermissions/

How to cite this article: Zou, Y. et al. Superior room-temperature ductility of typically brittle quasicrystals at small sizes. Nat. Commun. 7:12261 doi: 10.1038/ncomms12261 (2016).

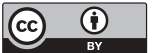

This work is licensed under a Creative Commons Attribution 4.0 International License. The images or other third party material in this article are included in the article's Creative Commons license, unless indicated otherwise in the credit line; if the material is not included under the Creative Commons license, users will need to obtain permission from the license holder to reproduce the material. To view a copy of this license, visit http://creativecommons.org/licenses/by/4.0/

(C) The Author(s) 2016 